\title{
Efeito da temperatura de calcinação nas propriedades de ossos bovinos para a fabricação de porcelana de ossos
}

\section{(Effect of calcination temperature on the properties of bovine bone ash for the fabrication of bone china)}

\author{
D. Gouvêa, S. Bernard, G. A. V. Alatrista, S. M. Tofolli \\ Departamento de Engenharia Metalúrgica e de Materiais, Escola Politécnica da Universidade de S. Paulo \\ Av. Prof. Mello Moraes 2463, Cidade Universitária, S. Paulo, SP 05508-900 \\ dgouvea@usp.br
}

\begin{abstract}
Resumo
Ossos bovinos calcinados são utilizados na fabricação de porcelana de ossos (bone china), consistindo em mais de $50 \%$ em peso das matérias-primas. A fabricação deste tipo especial de porcelana ainda é inédita no Brasil. Neste trabalho investigou-se a influência das etapas de calcinação, moagem e lavagem sobre as características físico-químicas das partículas de cinza de ossos. Os pós calcinados a temperaturas entre $700 \mathrm{e} 1000^{\circ} \mathrm{C}$ foram analisados quanto às suas distribuições de tamanho de partículas, composições químicas, composições de fases, densidades, área de superfície específica, grupos funcionais superficiais antes e depois de moídos e lavados. Os resultados indicam que os ossos processados nas temperaturas de 700, 800900 e $1000{ }^{\circ} \mathrm{C}$ apresentam grande homogeneidade tanto química (composição química e mobilidade eletroforética) como física (tamanhos de partículas e composição de fases) antes e depois da moagem e lavagem, Contudo, apresentam um leve aumento no tamanho médio de partículas com o aumento de temperatura de calcinação. Outro fato relevante na qualidade da superfície do pó obtido é que estes devem ser calcinados sob atmosferas oxidantes para eliminação de espécies adsorvidas que podem prejudicar a formação de barbotinas estáveis.

Palavras-chave: cinza de ossos, porcelana de ossos, bone china.
\end{abstract}

\begin{abstract}
Calcined bovine bones comprise the main raw material for the fabrication of Bone China (more than 50 wt.\%). This special kind of porcelain has never been produced in Brazil. In this paper, the influence of the following preparation steps: firing, grinding, and washing of the bones, on the physicochemical characteristics of the obtained bone ash particles was investigated. Powders calcined at temperatures between 700 and $1000{ }^{\circ} \mathrm{C}$, washed and ground, were analyzed in terms of their particle size distribution, chemical composition (X-ray fluorescence), density (He picnometry), specific surface area (BET method), infrared spectroscopy (FTIRDRIFT), and also in a scanning electron microscopy. The results indicated that bones processed at different temperatures exhibit great homogeneity either in chemical terms (chemical composition and electrophoretic mobility) or in physical terms (particle size and phase composition), show slight increase in particle size with increasing firing temperatures and must be calcined under oxidizing atmospheres in order to develop good processing characteristics.
\end{abstract}

Keywords: bone china, bone ash .

\section{INTRODUÇÃO}

Osso bovino calcinado $(\mathrm{OBC})$ é a principal matéria-prima utilizada na fabricação de um tipo especial de porcelana chamada de porcelana de ossos ou "bone china", que é um produto de grande resistência ao impacto, translucidez e alvura [1]. Fabricada com uma grande quantidade de ossos calcinados, cerca de $50 \%$ em peso, mas também podendo conter em sua formulação caulim, feldspato e quartzo, a porcelana de ossos é produzida em poucos países no mundo devido à disponibilidade da matéria-prima e também às dificuldades técnicas relacionadas ao processamento do produto, que está na maioria das vezes ligada ao processamento do OBC. A química coloidal envolvida na preparação do osso calcinado é bastante diferente daquela das argilas e caulins. Além disto, existe certa controvérsia a respeito da melhor temperatura de calcinação e das condições de moagem e lavagem do osso para a posterior preparação de barbotinas. Poucos trabalhos têm tratado do assunto, contudo Cooper [2], em publicação realizada a cerca de dez anos, enfatiza a importância do processamento de ossos bovinos com a finalidade de fabricação de bone china. Os resultados apresentados demonstram que a lavagem, o envelhecimento e a escolha da temperatura de calcinação são de fundamental importância para a qualidade do OBC. Resultados apresentados no trabalho indicam que o tamanho médio de partícula do $\mathrm{OBC}$ utilizado para a fabricação industrial deve ser cerca de $14 \mu \mathrm{m}$, pois neste tamanho existe pequena influência da temperatura de calcinação. Contudo, nenhuma justificativa foi abordada, como por exemplo, 
o empacotamento ou a físico-química da superfície das partículas. Cooper [2] descreve uma situação ocorrida na Inglaterra nos anos 70 onde houve um grande problema de produção ligado à qualidade do $\mathrm{OBC}$. Nenhuma análise tradicional, como difração de raios $\mathrm{X}$ e análise química, foi capaz de diferenciar os OBC "bons" dos "ruins". A técnica de espectroscopia no infravermelho esclareceu o problema, onde se podiam identificar grupos cianetos na superfície dos $\mathrm{OBC}$ que surgiam devido à calcinação em inadequada. Do ponto de vista da queima, a eliminação do problema ocorre com calcinação dos ossos bovinos a temperaturas superiores a $1000{ }^{\circ} \mathrm{C}$. Mais uma vez nenhuma a justificativa experimental foi apresentada. Outros autores sugerem que os produtores de porcelana de ossos utilizariam partículas bastante inferiores a $14 \mu \mathrm{m}$ com sucesso [3]. Concluem também que o tamanho de partícula pode afetar a velocidade de colagem, como deveria ser esperado, aumentando a velocidade de colagem para OBC com tamanhos médios de partículas maiores. Porém, nenhuma avaliação sistemática foi realizada e nenhuma citação de trabalhos anteriores foi apresentada nas duas publicações que pudesse evidenciar a importância da calcinação, da moagem e da lavagem dos ossos bovinos.

O objetivo deste trabalho foi avaliar sistematicamente a calcinação, moagem e lavagem dos ossos bovinos através da análise do tamanho médio e do estado de agregação das partículas, da variação da composição química, da densidade, da área de superfície específica e de aspectos físico-químicos dos grupos funcionais da superfície dos pós. Para atingir este fim, foram utilizadas as seguintes técnicas de caracterização: difração de raios X (XRD), fluorescência de raios $\mathrm{X}(\mathrm{XRF})$, espectroscopia no infravermelho com assessório de refletância difusa (FTIR-DRIFT), picnometria de He, microscopia eletrônica de varredura (SEM), mobilidade eletroforética dinâmica (ESA) e medidas de área de superfície específica pelo método BET.

\section{MATERIAIS E MÉTODOS}

Ossos bovinos foram lavados em autoclave para a eliminação do sangue e da maior parte da gordura associada, como descrito em trabalho anterior [4]. O osso bruto então foi seco em estufa a $100{ }^{\circ} \mathrm{C}$ e submetido à calcinação nas temperaturas de $700,800,900$ e $1000{ }^{\circ} \mathrm{C}$ por um período de $1 \mathrm{~h}$ em forno tipo mufla com introdução de ar atmosférico através de uma bomba. As condições de lavagem na autoclave e as temperaturas de calcinação foram estabelecidas em trabalho prévio [5]. Uma vez calcinados, os ossos foram moídos em almofariz de ágata, em seguida em moinho de bolas com jarro e elementos de porcelana por 24 h. Foi utilizando água destilada como fluído de moagem. Em seguida o material foi filtrado e lavado abundantemente com água destilada.

Os materiais foram então submetidos às análises de caracterização. As análises de distribuição granulométrica foram obtidas com um equipamento Mastersizer $\mathrm{S}$ da Malvern Instruments e realizadas em álcool isopropílico, com velocidade de bomba de $2500 \mathrm{rpm}$ e tratamento em ultra-som durante $1 \mathrm{~min}$. As análises de área de superfície específica (ASE) foram feitas com equipamento Gemini III 2375, Micromeritics. As amostras para medidas de ASE foram pré-tratadas em vácuo durante $12 \mathrm{~h}$ a $350^{\circ} \mathrm{C}$. Nas análises de espectroscopia no infravermelho (FTIR) foi empregado o equipamento Magna 560 com célula de refletância difusa - DRIFT da Nicolet Instr. Corp.. As amostras não foram submetidas a qualquer tratamento prévio e os espectros de FTIR foram obtidos diretamente na amostra e sem diluição em $\mathrm{KBr}$. As características ácidobásicas da superfície dos pós calcinados forma analisadas através de medidas de mobilidade eletroforética dinâmica em um aparelho ESA-8000. Matec Appl. Sci.. As dispersões de osso calcinado e moído com concentração volumétrica de $1 \%$ em água foram tituladas com solução de ácido nítrico $2 \mathrm{M}$ no intervalo de $\mathrm{pH}$ de 11 a 3 . Vale enfatizar que todas as dispersões apresentaram $\mathrm{pH}$ próximo de 11 durante a preparação inicial. A determinação da estrutura cristalina do OBC foi feita por difração de raios X, Philips X'PERT MPD, com radiação $\mathrm{k}_{\alpha}$ do cobre, na rotina $\theta / 2 \theta$ de 2 a $90^{\circ}$, com passo de $0,02^{\circ}$ e tempo de permanência de $1 \mathrm{~s}$. Para a observação do pó de osso calcinado foi usado microscópio eletrônico de varredura Philips XL-30, com filamento de tungstênio, tensão de aceleração de $20 \mathrm{kV}$. As partículas foram dispersas em um porta-amostra metálico através de uma solução aquosa e um filme condutor de $\mathrm{Au}$ foi depositado para contato elétrico.

\section{RESULTADOS E DISCUSSÃO}

As curvas de distribuição granulométrica do OBC tratado termicamente em diferentes temperaturas e desagregados em almofariz de ágata (Fig. 1) ou moídos e lavados (Fig. 2) demonstram uma nítida diferença do tamanho das partículas entre os dois procedimentos. Contudo, não há uma diferença significativa as diferentes temperaturas de calcinação em cada processo. Os tamanhos médios dos pós moídos e lavados obtidos a partir da distribuição granulométrica foram: 2,7, 2,6, 2,6 e 3,3 $\mu \mathrm{m}$, respectivamente para as temperaturas de $700,800,900$ e $1000^{\circ} \mathrm{C}$. Estes resultados contradizem os

Tabela I - Área de superfície específica e densidade do osso calcinado em diferentes temperaturas e o cálculo do tamanho de partícula, $\mathrm{D}_{\mathrm{BET}}$

TTable I - Specific surface area and density of bone calcined at different temperatures and the calculated mean particle size, $\left.D_{B E T}\right]$

\begin{tabular}{cccc}
\hline $\begin{array}{c}\text { Temperatura de } \\
\text { calcinação }\left({ }^{\circ} \mathrm{C}\right)\end{array}$ & $\begin{array}{c}\text { ASE } \\
\left(\mathrm{m}^{2} / \mathrm{g}\right)\end{array}$ & $\begin{array}{c}\text { Densidade } \\
\left(\mathrm{g} / \mathrm{cm}^{3}\right)\end{array}$ & $\begin{array}{c}\mathrm{D}_{\mathrm{BET}} \\
(\mu \mathrm{m})\end{array}$ \\
\hline 700 & 5,09 & 2,91 & 0,40 \\
800 & 4,57 & 2,51 & 0,52 \\
900 & 4,41 & 2,52 & 0,54 \\
1000 & 2,56 & 2,52 & 0,93 \\
\hline
\end{tabular}




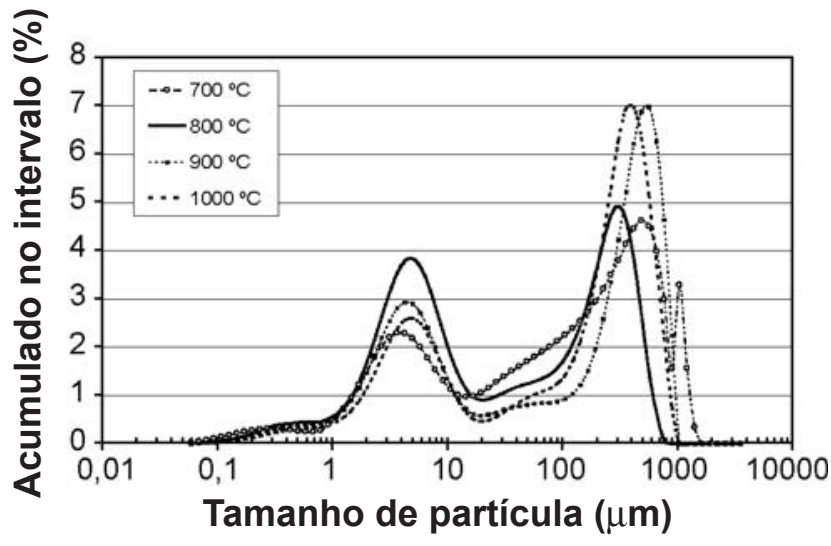

Figura 1: Distribuição de tamanho de partículas do osso calcinado a diferentes temperaturas e sem moagem e lavagem.

[Figure 1: Particle size distribution of bovine bone calcined at different temperatures before grinding and washing.]

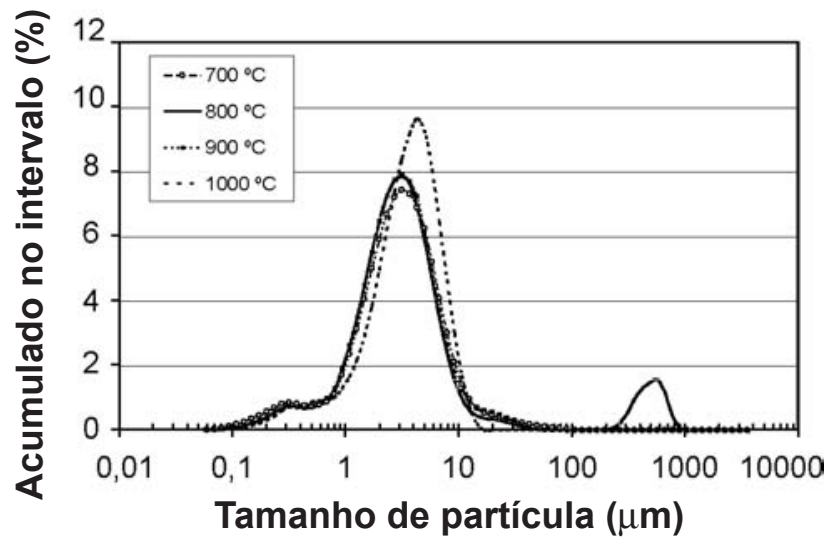

Figura 2: Distribuição de tamanho de partículas do osso calcinado em diferentes temperaturas, moído em moinho de bolas e lavados. [Figure 2: Particle size distribution of bovine bone calcined at different temperatures after grinding and washing.]

apresentados na literatura que relatam que a distribuição granulométrica é fortemente afetada pela temperatura de calcinação [2]. Medidas de densidade por picnometria de He e de área de superfície específica por adsorção de $\mathrm{N}_{2}$, método BET, permitem verificar uma evolução semelhante do tamanho médio de partículas para o pó calcinado, moído e lavado (Tabela I). Os resultados de tamanho médio calculado a partir destas técnicas são menores que aqueles obtidos através da distribuição granulométrica devido ao estado de aglomeração do material calcinado que não foi totalmente restituído no processo de moagem.

A observação do material através de microscopia eletrônica de varredura apresenta resultados bastante compatíveis com aqueles obtidos pelos ensaios de tamanho de partículas de ASE e densidade (Fig. 3). O material apresenta grande homogeneidade apesar de sua origem natural. As partículas são equiaxiais e não foi observado nenhum resíduo ou contaminação.

Nenhuma diferença significativa de mudança de fase
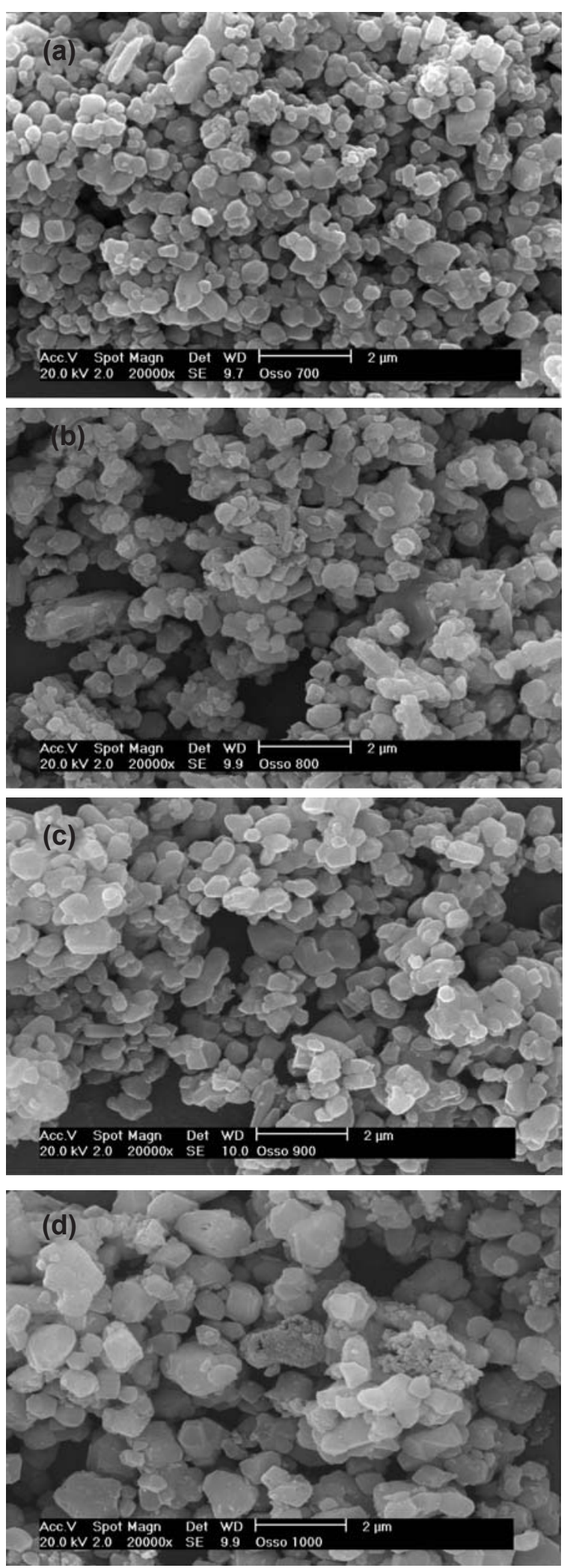

Figura 3: Micrografias obtidas por microscopia eletrônica de varredura da evolução do tamanho das partículas de osso calcinado nas temperaturas (a) 700, (b) 800 , (c) 900 e (d) $1000{ }^{\circ} \mathrm{C}$.

[Figure 3: SEM micrographs of bone particles calcined at different temperatures (a) 700, (b) 800, (c) 900 and (d) $1000^{\circ} \mathrm{C}$.] 
foi observada durante a moagem e lavagem (Fig. 4). A moagem e a lavagem parecem não ter efeito significativo na estabilidade da hidroxiapatita como observado em trabalhos anteriores $[2,3]$. A única diferença notada foi a presença de uma pequena quantidade de $\mathrm{CaO}$ para os materiais calcinados a $1000{ }^{\circ} \mathrm{C}$. A pequena quantidade de $\mathrm{CaO}$ não deve interferir de forma significativa na produção de bone china, contudo pode modificar as condições das dispersões. Estudos posteriores serão realizados para a verificação das condições de dispersão das barbotinas preparadas com OBC calcinados a em diferentes temperaturas.

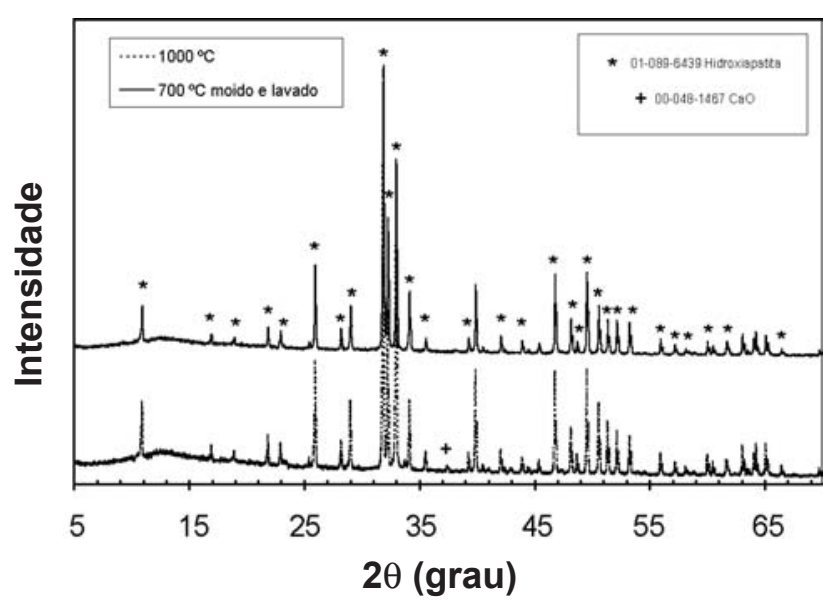

Figura 4: Padrões de difração de raios $\mathrm{X}$ do osso calcinado a $1000{ }^{\circ} \mathrm{C}$ e calcinado a $700^{\circ} \mathrm{C}$, moído e lavado.

[Figure 4: XRD patterns of bone calcined at $1000{ }^{\circ} \mathrm{C}$ before grinding and washing and calcined at $700^{\circ} \mathrm{C}$ after grinding and washing.]
Os resultados de análise química revelam uma grande homogeneidade da composição entre as diferentes temperaturas de calcinação (Tabela II) para os ossos calcinados e para os ossos moídos e lavados. Mais uma vez os resultados são diferentes daqueles obtidos por outros autores [5]. O teor de óxido de ferro nos materiais é muito baixo e não foi detectado em nenhum dos pós preparados neste trabalho, confirmando que a lavagem em autoclave é um método bastante eficiente para a retirada dos resíduos de sangue. O óxido de ferro, associado ao $\mathrm{TiO}_{2}$, é responsável pela cor levemente marfim da porcelana de osso quando queimada em atmosfera oxidante [1]. O maior efeito da moagem e lavagem dos ossos está associado à dissolução dos sais de potássio e sódio e a contaminação com alumina e sílica proveniente dos elementos de moagem [2,4]. Os resultados de análise química demonstram que, após a lavagem, os materiais têm reduzidos os resíduos de $\mathrm{Na}_{2} \mathrm{O}$ e $\mathrm{K}_{2} \mathrm{O}$. Contudo, as diferenças são pouco significativas e não devem afetar o sistema, por exemplo, durante a preparação de uma dispersão. Os efeitos de estabilidade das dispersões, do ponto de vista de envelhecimento do material, parecem estar mais ligados a fenômenos da físico-química de superfície do material do que propriamente da composição química global. O envelhecimento e a estabilidade das dispersões parece estar ligado às condições de calcinação dos ossos, como atmosfera do forno, e da exposição do osso úmido após a moagem à atmosfera [2, 3].

Análises das características da físico-química de superfície de óxidos cerâmicos são possíveis de serem estudadas através de técnicas simples como a determinação da mobilidade eletroforética (ESA) e de análise FTIRDRIFT [6]. Análises realizadas no OBC moído e lavado demonstram também que, nas condições de preparação

Tabela II - Análise química obtida através de fluorescência de raios X das amostras de ossos calcinados a diferentes lavadas ou não. [Table II - XRF chemical analysis of bone calcined at different temperatures before and after grinding and washing.]

\begin{tabular}{|c|c|c|c|c|c|c|c|c|}
\hline$\frac{\text { amostra }}{\text { Óxido (\%) }}$ & $700^{\circ} \mathrm{C}$ & $800^{\circ} \mathrm{C}$ & $900^{\circ} \mathrm{C}$ & $1000^{\circ} \mathrm{C}$ & $\begin{array}{c}700^{\circ} \mathrm{C} \\
\text { moído } \\
\text { e lavado }\end{array}$ & $\begin{array}{c}800{ }^{\circ} \mathrm{C} \\
\text { moído } \\
\text { e lavado }\end{array}$ & $\begin{array}{c}900{ }^{\circ} \mathrm{C} \\
\text { moído } \\
\text { e lavado }\end{array}$ & $\begin{array}{c}1000{ }^{\circ} \mathrm{C} \\
\text { moído } \\
\text { e lavado }\end{array}$ \\
\hline $\mathrm{CaO}$ & 55,3 & 55,3 & 55,3 & 55,6 & 54,6 & 54,6 & 55,0 & 55,0 \\
\hline $\mathrm{P}_{2} \mathrm{O}_{5}$ & 41,9 & 42,0 & 42,1 & 41,7 & 42,7 & 42,8 & 41,9 & 42,6 \\
\hline $\mathrm{Na}_{2} \mathrm{O}$ & 1,33 & 1,25 & 1,10 & 1,11 & 1,10 & 0,97 & 1,20 & 0,74 \\
\hline $\mathrm{MgO}$ & 1,11 & 1,11 & 1,12 & 1,13 & 1,11 & 1,12 & 1,34 & 1,12 \\
\hline $\mathrm{Al}_{2} \mathrm{O}_{3}$ & 0,01 & $<<$ & n.i. & n.i. & 0,19 & 0,24 & 0,18 & 0,21 \\
\hline $\mathrm{SiO}_{2}^{3}$ & 0,02 & 0,01 & n.i. & n.i. & 0,04 & 0,06 & 0,04 & 0,02 \\
\hline $\mathrm{SO}_{3}^{2}$ & 0,13 & 0,16 & 0,18 & 0,18 & 0,09 & 0,10 & 0,16 & 0,18 \\
\hline $\mathrm{Cl}^{3}$ & 0,10 & 0,13 & 0,07 & 0,06 & 0,07 & 0,07 & 0,06 & 0,06 \\
\hline $\mathrm{K}_{2} \mathrm{O}$ & 0,40 & 0,03 & 0,02 & 0,02 & 0,02 & 0,02 & 0,01 & n.i. \\
\hline $\mathrm{ZnO}$ & 0,02 & 0,02 & 0,02 & 0,02 & 0,02 & 0,02 & 0,01 & 0,02 \\
\hline $\mathrm{SrO}$ & 0,06 & 0,06 & 0,06 & 0,07 & 0,06 & 0,06 & 0,06 & 0,06 \\
\hline $\mathrm{BaO}$ & 0,02 & $<<$ & n.i. & 0,10 & 0,03 & 0,03 & 0,04 & n.i. \\
\hline
\end{tabular}

$<<=$ traços e n.i. = não identificado 
deste trabalho, as superfícies exibem pouca diferença nas suas características (Figs. 5 e 6). As análises de mobilidade eletroforética dinâmica em função do pH (ESA - Fig. 5) mostram que o ponto isoelétrico flutua entre 10,3 e 9,4 com a mudança da temperatura de calcinação e pouco efeito pode ter sobre a estabilidade das dispersões, já que nestas condições os dispersantes de sais derivados de ácido poliacrílico e o próprio silicato de sódio apresentam uma ótima funcionalidade [7]. Isto deve permitir a fabricação de dispersões estáveis neste sistema com estes tipos de dispersantes. A interação do $\mathrm{OBC}$ com as outras matériasprimas que compõem o sistema bone china, como caulim e feldspato, serÁ assunto de uma próxima investigação.

Os resultados de FTIR - DRIFT confirmam as observações realizadas através das análises de ESA (Fig. 6). Pouca diferença é observada entre as amostras analisadas. Os

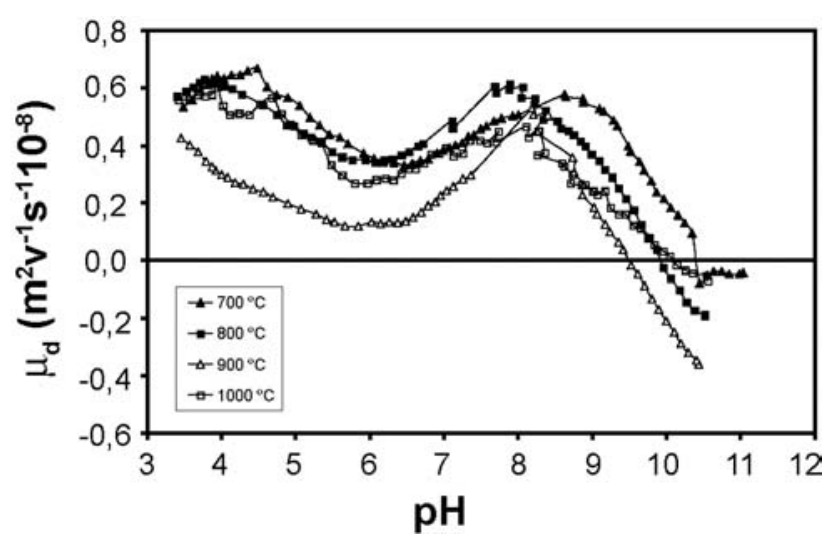

Figura 5: Mobilidade eletrocinética dinâmica como função do pH para o $\mathrm{OBC}$ em diferentes temperaturas, moído e lavado.

[Figure 5: Electrokinetic dynamic mobility versus pH of bone calcined at different temperatures.]

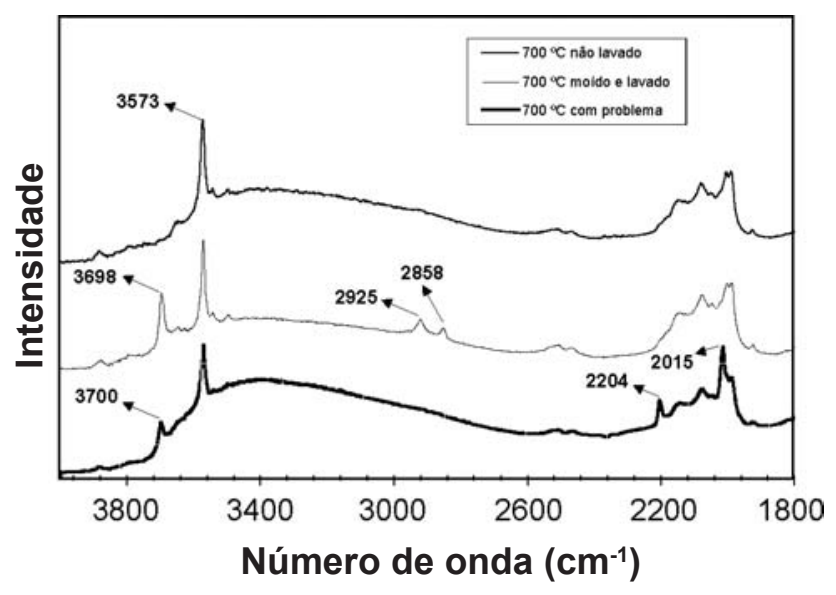

Figura 6: DRIFT do osso calcinado a $700{ }^{\circ} \mathrm{C}$ com diferentes condições de processamento e comparação com osso com problemas durante a preparação de dispersões.

[Figure 6: DRIFT spectra of the calcined bone at $700{ }^{\circ} \mathrm{C}$ after and before grinding and washing and bone with problem during dispersion preparation.] espectros de DRIFT obtidos apresentam bandas de absorção que evidenciam a superfície do pó analisado, diferentemente da técnica em transmissão com pastilha de $\mathrm{KBr}$ que evidencia as características das ligações químicas da rede cristalina. Desta forma, os espectros são ligeiramente diferentes dos encontrados na literatura [2]. As mudanças notadas nos espectros DRIFT foram referentes à hidratação da superfície sendo que são observadas as bandas a $3573 \mathrm{~cm}^{-1}$ para todas as amostras e após hidratação surge a banda a $3700 \mathrm{~cm}^{-1}$, que é associada à vibração de estiramento de hidroxilas da superfície [6]. Picos de baixa intensidade com vibrações a 2925 e $2858 \mathrm{~cm}^{-1}$ foram também observados após a lavagem. Estes picos estão normalmente associados à ligação $\mathrm{C}-\mathrm{H}$ de compostos orgânicos. Como os mesmos picos foram encontrados nos pós calcinados a $1000{ }^{\circ} \mathrm{C}$ e somente após moagem e lavagem devem estar provavelmente associados a contaminação devido a resíduos de dispersantes presentes nos elementos de moagem e não a resíduos orgânicos do osso. Como a técnica de FTIR-DRIFT é muito sensível, podem-se observar poucas moléculas de poliacrilatos adsorvidas à superfície e que são muito utilizadas nos procedimentos de laboratório. Contudo, materiais que foram calcinados sem a introdução de ar no forno apresentaram picos a 2204 e $2015 \mathrm{~cm}^{-1}$ que não existem nos materiais calcinados em atmosfera oxidante. Os materiais assim calcinados não formam dispersões estáveis e geram dificuldades na preparação de barbotinas. Trabalhos fazem referência a este comportamento e denominando esse material de "osso ruim" [2]. Os picos nos espectros de FTIR obtido por transmissão dos materiais foram a aproximadamente $2000 \mathrm{~cm}^{-1}$ [2], que não são equivalentes aos encontrados no presente trabalho e foram atribuídos à formação de cianetos na superfície dos ossos provenientes da queima incompleta do material orgânico contido nos ossos bovinos.

\section{CONCLUSÕES}

Ossos de bovinos calcinados (OBC) compõem grande parte da composição da porcelana bone china. Contudo, poucos estudos têm sido realizados no intuito de revelar as melhores condições de preparação do osso. Neste trabalho foi investigada a influência da temperatura de calcinação nas propriedades do OBC. Verificou-se que a temperatura de calcinação tem pouca influência sobre a granulometria e os aspectos físico-químicos de superfície para materiais moídos e lavados. O tamanho médio de partículas obtido por ensaios de difração laser foi de aproximadamente $3 \mu \mathrm{m}$. Contudo os resultados de tamanho médio obtidos a partir de medidas de área de superfície específica e densidade revelam que as partículas são menores com tamanho médio próximo de $0,5 \mu \mathrm{m}$. As análises físico-química da superfície realizadas através de medidas de ESA e DRIFT revelaram uma grande semelhança nas características da superfície para diferentes temperaturas de calcinação. A atmosfera do forno no momento da calcinação é muito mais importante que a temperatura em si, no intervalo de 700 a $1000{ }^{\circ} \mathrm{C}$. Atmosferas oxidantes são necessárias para impedir a formação de espécies adsorvidas nas superfícies do $\mathrm{OBC}$ e 
garantir as condições de preparação de dispersões estáveis. Os resultados obtidos demonstram ser possível obter OBC para uso na fabricação de porcelana a partir de temperaturas de $700{ }^{\circ} \mathrm{C}$. Contudo, estudos mais detalhados sobre a físicoquímica de superfície dos materiais preparados em diversas condições e a interação com outras matérias-primas deverão ser realizados para conclusões mais definitivas das condições de preparação de barbotinas para bone china.

\section{AGRADECIMENTOS}

Ao apoio financeiro da FAPESP (Projeto: Desenvolvimento do processo nacional para a fabricação de porcelana de ossos - bone china, Proc. 03/12721-2), CNPq (Projeto MACEPLAS, Edital Universal 01/2002, Proc. 475029/2003-8) e à École Nationale Supérieure de Chimie de Lille, França, pela concessão da bolsa de estagiário a Sebastian Bernard para execução do estágio de formatura e também à CAPES pela concessão da bolsa de mestrado.

\section{REFERÊNCIAS}

[1] P. Rado, An Introduction to the Technology of Pottery, $2^{\text {nd }}$ Ed., The Institute of Ceramics, Pergamon Press, Oxford (1988).

[2] J. J. Cooper, Bone for Bone China, Brit. Ceram. Trans. J. 94, 4 (1995) 165.

[3] D. Basnett, P. J. Cartwright, Brit. Ceram. Trans. J. 88, 5 (1989) 191.

[4] R.Y. Miyahara, D. Gouvêa, S.M. Toffoli, Cerâmica 53, 327 (2007) 127.

[5] R. Bragança, C.P. Bergmann, Cerâmica 52, 322 (2006) 210.

[6] G. J. Pereira, R. H. R.Castro, P. Hidalgo, D. Gouvêa, Appl. Surf. Sci. 195 (2002) 283.

[7] R. H. R. Castro, B. B. S. Murad, D. Gouvêa, Ceram. Int. 30 (2004) 2215.

(Rec. 28/02/2007, Ac. 17/08/2007) 